\title{
Evaluation of factors that affecting mortality in urgent redo-laparotomies
}

\author{
Erdal Uysal ${ }^{1 *}$, Serkan Kadir Turel ${ }^{2}$, Efe Sezgin ${ }^{3}$
}

\begin{abstract}
Objective: The aim of this study was to investigate the factors that affect mortality in patients undergoing Urgent abdominal redo-laparatomies (UARLs), and to analyze the common indications and operative, demograhic, andclinical characteristics in UARLs.

Material and Methods: Our study was designed as a retrospective, observational and multi-centric study. A total of 155 patients from two separate clinics undergoing urgent, unplanned redo-laparatomy were included in the study. The data obtained from all clinics were collected and and the relations of the demographic, clinical and operational factors with the mortalities of primary surgery and multiple UARLs were analyzed.

Results: Mortality was observed in $42(27 \%)$ patients. The most frequent causes of mortality were sepsis and multi-system organ failure in $23(53.4 \%)$ patients. The relationships between the number of redo-laparotomy presence of blood transfusion, older age,classification of surgery and mortality were significant $(\mathrm{p}<0.05)$.

Conclusion: Sepsis and multi-organ failure were the most frequent cause of mortality in our study as well, with a rate of $53.4 \%$. No significant relationship was observed between mortality and initial surgery under emergency conditions and presence of comorbidity. Redolaparatomies inevitable in some clinical situations. Since multiple redolaparatomies are associated with mortality, it is necessary to avoid complications during initial surgery to reduce UARLs. Major surgery operation UARLs in elderly people should be avoided. Finally, the surgeon should also make the UARLs decision at the right time.
\end{abstract}

Key words: Urgent, redo-laparatomy, mortality

\section{Introduction}

Abdominal surgical interventions are applied for the treatment of benign and malignant diseases under urgent and elective conditions. Although laparascopic abdominal interventions are widely preferred currently, laparatomy has been used frequently as well. The term redo-laparatomy or re-laparatomy is used for the laparotomies performed within 60 days following the primary surgery. Redo-laparatomies may be performed urgently, electively, planned or unplanned (1).

In our study, redo-laparatomies performed within 10 days following the initial surgery were described as urgent redo-laparatomies. Urgent abdominal redolaparatomies (UARLs) have been conisdered as complicated and last option operations. The rates of mortality and morbidity of UARLs are quite high (2).

UARLs are generally performed due to the complications observed after the initial operation. The most common indications for UARLs include anastomosis leaks, intra-abdominal abscess, peritonitis, mechanical intestinal obstruction, intraabdominal hemorrhage, intestinal necrosis and intraabdominal organ injuries (2).

Patients indicated for UARLs generally have a critical health situation. UARLs are still the most important step of the treatment for these patients $(1,3)$. The mortality rates vary between 50 and $100 \%$ in cases with peritonitis that cannot be taken under control, namely those with spesis and multiple system organ failure $(4,5)$.

UARLs may be peformed as on-demand surgery and planned. In planned UARLs, laparatomy is performed with pre-determined time intervals and thus the clinical situation is considered (6).

Both planned and on-demand surgeries are performed in the clinical centers in our study. UARLs may be performed once or multiple times according to clinical need.

Received 22-05-2017 Accepted 28-05-2017 Available Online 31-05-2017

1 Sanko University, Medical Faculty, Dept. of General Surgery, Gaziantep, TR

2 Afyon State Hospital, Dept. of General Surgery, Afyon, TR

3 Izmir Institute of Technology, Engineering Faculty, Department of Food Engineering, Izmir, TR

* Corresponding Author: Erdal Uysal E-mail: drerdaluysal@hotmail.com Phone: + 903422116569

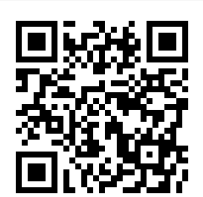


Although UARLs are widely used, studies investigating the factors that affect mortality in patients undergoing UARLs are limited in number. We believe that a good analysis of the factors that affect mortality and avoiding these factors would reduce mortality rates after UARLs. The aim of this study was to investigate the factors that affect mortality in patients undergoing UARLs, and to analyze the common indications and operativedemograhic-clinical characteristics in UARLs.

\section{Material and Methods}

Our study was designed as a retrospective, observational and multi-centric study. A total of 155 patients undergoing urgent, unplanned redolaparatomy among 2,830 patients undergoing abdominal surgery between January 2005 and July 2016, were included in the study. The participants were those who had undergone redo-laparatomy within 10 days following the initial surgery.

Two separate clinical centers were included in the study. The data obtained from all clinics were collected in a common electronic database. The data of the patients were obtained from the files and computer records according to the study protocol prepared previously by the study group.

In order to homogenize the data, the inclusion criteria were kept limited. Patients undergoing thoracoabdominal surgery, laparascopic surgery as the primary surgery and transplantations, those who had not undergone urgent redo-laparatomy, obstetric and pediatric patients, and patients treated via only interventional radiology or endoscopic interventions were excluded from the study.

Patients undergoing interventional radiology or endoscopic interventions prior to or after UARLs were not included either. Besides, superficial procedures not undergoing anesthesia were excluded from the study. The demographic characteristics, laboratory studies, radiographic examinations, operative reports, presence of concomitant adherent diseases such as malignancies, primary surgeries, postoperative complications, presence of multiple system organ failure, causes of mortality, clinical situations related to UAR, blood transfusions, duration of stay in emergency unit, duration of hospital stay, the interval between the laparotomies and the number of UARLs performed, were investigated.

The relations of the demographic and clinical factors with the mortalities of primary surgery and multiple UARLs were analyzed. The decision of UARLs were made according to the clinical situation of the patient, to radiological imaging results and to the laboratory findings.

The patients were followed-up for a minimum of 2 months following the operation, and mortalities in this period were included in the analysis. Information on the operations and follow-up were provided by the surgeons who performed the primary operations and UARLs when necessary. The operations were performed by general surgeons experienced in gastrointestinal system surgeries.

The centers involved in the study are high volume and provide intensive care and surgery services at similar levels. General surgeons at centers participating in the study have a similar approach to primary and redo surgical decisions. All centers use the same diagnostic tools in UARLs decision. The antibiotics and inotropic agents administered to patients were determined in a multi-disciplinary manner according to the needs of the patients. This study is registered at researchregistry.com by the number ID researchregistry 1778

\section{Statistical analysis}

All statistical analyses are conducted by a statistician. Distribution of categorical factors (such as comorbidities, presence of blood transfusion, etc.) among mortality and no-mortality groups were compared by Chi-square tests. Mortality and nomortality group means for the continuous variables (such as age) were compared by T-test. P-values less than 0.05 were considered as statistically significant. All statistical analyses were conducted by SAS/STAT version 9.3 (SAS Institute, Inc, Cary, North Carolina, USA)

\section{Results}

A total of 155 patients undergoing UARL were included in the study. The median patient age was 63 $(49,76)$. The ratio of female to male was $61 / 94(0.64)$. The initial operations were urgent in $74 \%$ of the patients. The number of redo-laparatomies was found to be $1.18 \pm 0.58$ per patient. The median days $(25 \%, 75 \%)$ interval to first redo-laparatomywas 5 $(2,7)$. The number of total comorbidity was 104 . The most frequent comorbidity was heart disease with 50 (44\%) patients (Table 1). The most frequent cause of UARLs was peritonitis in $62(40 \%)$ patients, and the second most frequent cause was abscess in $26(16.7 \%)$ patients. UARLs were performed with more than one indication in $17(10.9 \%)$ of the patients. The decision of UARL was most frequently made via Physical examination in $139(56 \%)$ patients. In $93(60 \%)$ of the patients, UARL wad decided using more than one tool (Table 2).

The site of index surgery included the Biliary tract and gallbladder in $44(28 \%)$ patients, colo-rectal in 34 $(22 \%)$ and the small bowel in $31(20 \%)$ patients, respectively. B group operations (Major surgery) were most frequently performed according to the classification of surgery in $114(74 \%)$ patients. 137 $(89 \%)$ patients had only one UARL, whereas $4(3 \%)$ patients had over 3 redo-laporatomy (Table 3 ) 
Table 1: Patient Characteristics

\begin{tabular}{|c|c|c|}
\hline Total Patients $n=155$ & \multicolumn{2}{|l|}{$\%$} \\
\hline $\operatorname{Sex}(\mathrm{F} / \mathrm{M})$ & $61 / 94$ & \\
\hline Age & $63(49,76)$ & \\
\hline Emergency Operation & 74 & \\
\hline Number of redo-laparatomy & $1.18 \pm 0.58$ & \\
\hline Median days Interval to first redo-laparatomy $(25 \%, 75 \%)$ & $5(2,7)$ & \\
\hline Median days Hospital stay $(25 \%, 75 \%)$ & $12(9,19)$ & \\
\hline Median days ICU stay $(25 \%, 75 \%)$ & $9(4,13)$ & \\
\hline Comorbidite & $\mathbf{n}$ & $\%$ \\
\hline - Heart disease & 50 & 44 \\
\hline - Pulmonary disease & 13 & 11,4 \\
\hline - Renal disease & 14 & 12,3 \\
\hline - Diabetes & 19 & 17 \\
\hline - $\quad$ Others & 18 & 15,8 \\
\hline Total (n) & 104 & \\
\hline Causes of death & & \\
\hline - $\quad$ Sepsis and MOF & 23 & 53,4 \\
\hline - Intraabdominal hemorrhage & 4 & 9,3 \\
\hline - $\quad$ Respiratory failure and pneumonia & 8 & 18,6 \\
\hline - Cardiac & 3 & 6,9 \\
\hline - Unknown & 4 & 11,6 \\
\hline Total (n) & 42 & \\
\hline
\end{tabular}

M: male, F: female, ICU: intensive care unit, MOF: Multiorgan Failure,

Comorbidite others: Autoimmune diseases, thyroid disease, hematological diseases, multiple comorbidite

Table 2: Indications of urgent Redo-laparotomy and decision tool

\begin{tabular}{lcc}
\hline Patients $(\mathbf{n}=\mathbf{1 5 5})$ & $\mathrm{n}$ & $\%$ \\
Indications & & 7 \\
- Anastomotic leak & 11 & 13,5 \\
- Intra-abdominal hemorrhage & 21 & 40 \\
- Peritonitis & 62 & 16,7 \\
- Abscess & 26 & 13,5 \\
- Intestinal obstruction & 21 & 3,8 \\
- Bowel necrosis & 6 & 4,5 \\
- Abdominal dehiscence & 7 & 11,6 \\
- Other & 18 & 110,6 \\
Total & 172 & 34.2 \\
Decision tool & & 56 \\
Computed tomography/ultrasonography & 85 & 6.4 \\
Physical examination & 139 & 3.2 \\
Purulent discharge (wound) /drained content & 16 & $\mathbf{1 0 0}$ \\
Others & 8 & $\mathbf{2 4 8}$ \\
Total
\end{tabular}

Decision tool others: Multiple organ failure, positive blood culture, roentgenography, unexplained sepsis 
Table 3: Operational characteristics

\begin{tabular}{|c|c|c|}
\hline Patients (n:155) & $\mathrm{n}$ & $\%$ \\
\hline \multicolumn{3}{|l|}{ Site of index Surgery } \\
\hline - Pancreas & 3 & 2 \\
\hline - Colo-rectal & 34 & 22 \\
\hline - Small Bowel & 31 & 20 \\
\hline - Biliary tract and gallbladder & 44 & 28 \\
\hline - Gastro-duedonal & 17 & 11 \\
\hline - $\quad$ Liver & 8 & 5 \\
\hline - $\quad$ Appendix & 16 & 10 \\
\hline - Spleen & 2 & 1 \\
\hline \multicolumn{3}{|l|}{ Classification of surgery* } \\
\hline - $\mathrm{A}$ & 23 & 15 \\
\hline - $\mathrm{B}$ & 114 & 74 \\
\hline - $\mathrm{C}$ & 18 & 12 \\
\hline \multicolumn{3}{|l|}{ The number of redo-laparotomy } \\
\hline - 1 & 137 & 89 \\
\hline - 2 & 11 & 7 \\
\hline - 3 & 3 & 2 \\
\hline - $>3$ & 4 & 3 \\
\hline \multicolumn{3}{|l|}{ Initial operation } \\
\hline - Emergency & 115 & 74 \\
\hline - Electively & 40 & 26 \\
\hline
\end{tabular}

A group operation : Featured, major surgery and initiatives B group operation: Major surgery, C group operation: Medium-sized operations. (*Turkish Ministry of Health Annex- 9 List, 2015, classification of surgery list).

Mortality was observed in $42(27 \%)$ patients. The most frequent causes of mortality were sepsis and multi-system organ failure in $23(53.4 \%)$ patients. This was followed by Respiratory failure and pneumonia in $8(18.6 \%)$ patients. The relationship between the number of redo-laparotomy and mortality was found to be significant $(\mathrm{p}=0.04)$. Besides, a significant correlation was observed for blood transfusions, the number of redo-laparatomies, age, site of index surgery and classification of surgery $(\mathrm{p}<0.05)$.

No significant relationship was observed between comorbidity, elective or emergency operations, indication at redo-laparotomy and mortality $(\mathrm{p}>0.05)$.
No significant difference was observed between male and female patients with respect to mortality $(\mathrm{P}=0.85)$ (Table 4).

Primary abdominal closure was performed on 118 $(76 \%)$ patients subsequent to presumed source control. Secondary abdominal closure via mesh, and Bogota bag were performed on 37 (24\%) patients. 46 (29.6\%) patients recieved critical care support.

$28(18 \%)$ patients recieved ventilator support (over 48 hours). 30 (19.3\%) patients recieved total paranteral nutrition support. All patients received paranteral antibiotics. 
Table 4: Factors associated with mortality

Female

Comorbidite (n)

- Heart disease

- Pulmonary disease

- Renal disease

- Diabetes

- Others

Emergency/ Electively operations

Presence of blood transfusion

The number of blood transfusion

- 1 Unit

- 2 Unit

- 3 Unit

- 4 Unit

- $\quad>4$ Unit

Classification of surgery*

- A

- $\mathrm{B}$

- $\mathrm{C}$

The number of redo-laparotomy

- 1

- 2

- 3

- $>3$

Site of index Surgery

Pancreas

Colo-rectal

Small Bowel

Biliary tract and gallbladder

Gastro-duedonal

Liver

Appendix

Spleen

Indication at redo-laparotomy

Anastomotic leak

Intra-abdominal hemorrhage

Peritonitis

Abscess

Intestinal obstruction

Bowel necrosis

Abdominal dehiscence

Other

Age

Yes (n:42)
$16(38)$

$19(50)$

7 (18)

4 (10)

4 (10)

$12(30)$

$29 / 13$

36 (86)

7 (21)

$17(52)$

6 (18)

$2(6)$

$1(3)$

13 (31)

$29(69)$

$0(0)$

32 (76)

6 (14)

1 (2)

3 (7)

$3(7)$

9 (21)

$11(26)$

8 (19)

$10(24)$

$1(2)$

$0(0)$

$0(0)$

5 (12)

6 (14)

$19(45)$

$5(12)$

$6(14)$

$4(9)$

2 (5)

5 (12)

$71.5(61.0 / 81.0)$

\section{Mortality}

No (n:113)

45 (40)

p
0.84

0.06

$31(41)$

$6(8)$

$10(13)$

$15(20)$

$6(8)$

$86 / 27$

53 (47)

0.37

$<.0001$

0.29

$9(18)$

31 (61)

$4(8)$

1 (2)

6 (12)

10 (9)

$85(75)$

18 (16)

104 (92)

5 (4)

2 (2)

$1(0.9)$

0 (0)

25 (22)

20 (18)

$36(32)$

7 (6)

7 (6)

16 (14)

2 (2)

$6(5)$

15 (13)

43 (35)

21 (18)

15 (13)

2 (2)

5 (4)

$13(12)$

$60(45,73) \quad \mathbf{0 . 0 0 2}$

$5(2,7)$
0.04

0.0005

0.54

0.61
0.0002

Median days Interval to first redo-laparatomy

$4(2,6)$ $(25 \%, 75 \%)$

A group operation: Featured, major surgery and initiatives, B group operation: Major surgery C group operation: Medium-sized operations. (*Turkish Ministry of Health Annex- 9 List, 2015, classification of surgery list) The results were given as mean \pm standard deviation. Site of index Surgery others: Abdominal Wall, multiple site 


\section{Discussion}

UARLs are generally performed due to the complications observed following the initial operation. Anastamosis leaks, intra-abdominal abscess, peritonitis, mechanical intestinal obstruction and intraabdominal hemorrhage are the main indications for UARLs. The incidence of UARLs varies according to the initial operations. The incidence of UARLs has been reported as $1-4.4 \%$ in different studies $(2,7,8)$. In our study, the incidence of UARLs was $5 \%$.

Different clinical centers report different orders for the frequency of causes of UARLs. In the study of Unalp et al., the most frequent causes of UARLs were intestinal repair or anastamosis leaks in $51.85 \%$ of the patients, followed by intra-abdominal hemorrhage in $18.51 \%$ and intra-abdominal abscess in $9.87 \%$. Koirala $\mathrm{R}$ et al. , have reported intra-abdominal hemorrhage, intraabdominal abscess and collections as the most frequent causes of UARLs in $34.2 \%$ and $29.6 \%$ patients, respectively $(1,2)$. Koirala $\mathrm{R}$ et al. have related the high rate of inta-abdominal hemorrhage in their study to the high rate of hepatic and pancreatic surgeries (2). In our study, UARLs were most frequently performed due to peritonitis in $40 \%$ of the patients, intra-abdominal hemorrhage in $13.5 \%$, intra-abdominal abscess in $16.7 \%$ and mechanical intestinal obstruction in $13.5 \%$. Intraabdominal abscesses are common. Residual intraabdominal abscesses may be observed in gastrointestinal perforations or following the surgical treatment of acute appencitis. Intra-abdominal abscesses may additionally be observed in anastomosis leaks of the gastrointestinal system. The majority of intra-abdominal abscesses may be drained by interventional radiology. However, in presence of diffuse intra-abdominal abscess, in situations where generalized peritonitis accompanies the abscess and where drainegae is technically impossible, surgical drainage may be preferred. In our stuy, patients for whom intra-abdominal abscess drainage could not be performed by interventional radiology were evaluated. Thus, the rate of intra-abdominal abscess-related UARLs were found to be high. Intra-abdominal hemorrhages form the first order in patients who had undergone multiple UARLs in our study, with a rate of $13.5 \%$. The cause of increased intra-abdominal hemorrhage in initial UARLs may be related to insufficient hemostasis, impaired coagulation in patients, insufficient amount of coagulation factors and massive blood transfusion.

UARLs were most frequently performed following biliary tract and gallbladder surgery in our study, with a rate of $28 \%$. The most frequent causes of UARLs in biliary tract and gallbladder surgery were found to be intra-abdominal hemorrhage and biliary leaks, respectively. The high rate of initial biliary tract and gallbladder surgery was believed to be related to the increase in the number of UARLs. Furthermore, studies report high rates of intra-abdominal hemorrhage and anastomosis leaks following pancreas surgery $(9,10)$. Increased rates of UARLs may be related to the increased rate of complications. The most frequent causes of UARLs in patients undergoing colo-rectal surgery were found to be anastamosis leaks, intra-abdominal abescess and peritonitis, respectively. In a study evaluating relaparatomies, the colon was reported to be the major source of intra-abdominal infection leading to peritonitis (11). Anastomosis leaks, intra-abdominal abescess and peritonitis were mostly observed in patients undergoing colorectal surgery in our study.

UARL was performed due to anastomosis leak in $7 \%$ of the patients in our study. The most frequent anastomosis leaks were observed in colo-colic, colorectal, oesophago-jejunostomy and pancreaticojejunostomy anastomoses, respectively. The last anastomosis leak was observed in the anastomoses performed between the small intestines. Anastomosis leaks lead to generalized peritonitis, sepsis, fluid and electrolyte loss, multi-system organ failure, and may result in death. In some studies, high mortality rates have been reported following UARLs performed due to peritonitis. On the other hand, there are studies reporting reduced mortality rates following planned re-laparatomies since they provided effective irrigation and drainage (11-14). Consideration of UARLs has been suggested in the treatment of uncontrolled intra-abdominal infection and multisystem organ failure (15).

Mortality was mostly observed in relation to multisystem organ failure and sepsis developing following anastomosis leaks in our study. Despite multi-redolaparatomies performed on the patients, high mortality rates were encountered. In the study of Sautner $\mathrm{T}$ et al., re-laparatomies performed on patients with abdominal sepsis were reported to increase the inflammatory response, and the increased inflammatory response was reported to increase the mortality rates (12). In another study, re-laparatomy was reported to change the multi-system organ failure into an irreversible situation when the treatment to be performed with re-laparatomy was not properly selected (16). Purulant, fecal and biliary peritonitis may continue at a rate of $9-41 \%$ despite redolaparatomies (17). The reason for the sepsis and mortality that could not be controlled despite UARL in our study may be the peritonitis, increased inflammatory response and insufficient surgery in the first UARL.

In addition to the sufficient surgical treatment in UARL, the timing of the surgery is also important. A delayed surgical intervention on the intra-abdominal septic focus may lead to sepsis and multi-organ failure. Therefore, early diagnosis and treatment will reduce the mortality and morbidity $(7,18)$. Mortality rates may be reduced from $46 \%$ to $26.5 \%$ with early diagnosis and treatment (19). 
In our study, the Median interval to first redolaparatomy was $5(2,7)$ days in patients without mortality; it was $4(2,6)$ days in patients with mortality. The difference between the two groups was not statistically significant. Despite the advances in the surgical techniques and intensive care conditions, the mortality rates after UARLs have been reported to be as high as $61.5 \%$ (1). In a study evaluating UARLs, the mortality rate was found to be $33 \%$ (2). It was reported to be $37.03 \%$ in the study of Unalp et al. (1). In our study, the mortality rate was $27 \%$. Our findingis consistent with the findings in the literature.

Ching SS et al. have classified the risk of mortality in UARLs as low, moderate and high. Accordingly, wound separation was in the low risk group, and anastamosis leaks were in the high risk group (9). Unalp et al. have reported mesentery artery embolus, intestinal perforation and anastomosis failure in the high mortality risk group, and intra-abdominal infection and abscess in the moderate and low risk groups, respectively (1). Koirala $\mathrm{R}$ et al. have reported intra-abdominal hemorrhages as diseases with the highest mortality (2). In the same study, fecal fistula without evidence of anastomotic failure was reported to be the disease with the lowest mortality. In our study, sepsis and multi-system organ failure was the most common cause of mortality with a rate of $53.4 \%$. It was followed by respiratory failure and pneumonia with a rate of $18.6 \%$, and respiratory failure and intraabdominal hemorrhage at a rate of $9.3 \%$. However, no significant relationship was observed between the indication for redo-laparotomy and the mortality $(\mathrm{p}=0.54)$.

High mortality rates have been reported in UARLs performed subsequent to gastrointestinal system surgeries (20). Mortality rates were high among patients undergoing gastrointestinal system surgeries. Significant relationship was observed between the site of initial surgery and the mortality in our study $(\mathrm{p}=0.0005)$.

It has been reported that UARLs performed in unexplained sepsis resistant to medical treatment reduced the mortality. Holzheimer and Gathof have demonstrated that re-laparatomy reduced the mortality rates from $67 \%$ to $37.5 \%$ in persistent sepsis (21). However, it is impossible to determine the septic foci in all cases. Determining the septic focus is possible in only $17 \%$ of the patients (22). In our study, UARL was performed on $5(3.2 \%)$ patients due to unexplained resistant sepsis. The mortality rate in this group was $20 \%$. This rate constituted $2.3 \%$ of the total mortality.

A significant relationship has been reported between the number of redo-laparotomies and the mortality in previous studies. It has been reported in the study of Rygachev GP et al. that the mortality rates were significantly higher in multiple re-laparatomies compared to single re-laparatomies (23). In the study of Koirala R et al. , the mortality rates were reported to be $23.6 \%$ in single relaparatomies and $61.2 \%$ in multiple re-laparatomies (1). Rygachev GP et al. have found significant differences between single and multiple redo-laparatomies with regard to mortality rates (23). The patients may undergo multiple redolaparatomies due to an improper initial redolaparatomy. In our study, the mean number of UARLs in patients with mortality was $1.18 \pm 0.58$ per patient. The difference between the two groups was statistically significant $(\mathrm{p}=0.04)$. The presence of a residual infection, insufficient treatment in the initial UARL, insufficient managing of newly developed complications and reduced patient reserve may be responsible for the high mortality rates. The higher incidence of multi-organ failures in elderly patients supports this finding (24). Unalp et al. have reported the etiology rather than the number of redolaparotomies as the resposible factor for increased mortality (1).

Postoperative intra-abdominal hemorrhage is an important cause of UARLs. Bleeding may originate from a major vascular structure or a small vascular structure. Intra-abdominal hemorrhage may originate from the edges of the drain, the incision line, or from the upper or lower gastrointestinal system. UARLs may be necessary in large volume bleedings that impair the hemodynamics of the patient. Disseminated intravascular coagulopathy may also develop in delayed bleedings that necessitate recurrent blood transfusions. UARL may also be necessary for these patients (1). The rate of hemorrhage in abdominal surgeries has generally been reported to be between 0.9 and $4.7 \%$ (25). In another study, the rate of postoperative intra-abominal hemorrhages was reported to be between 3.3 and $19 \%$ in patients undergoing UARL $(26,27)$. The rate of hemorrhage following abdominal surgical interventions was reported to be $0.1 \%$ in the study of Kononov AG et al. The reason for the low rate of intra-abdominal hemorrhage in the study of Kononov AG et al. was related to the early diagnosis and good preoperative preparation of the patients with the risk of bleeding (28). Rate of UARLs was found to be $1 \%$ in our study, since intra-abdominal hemorrhage was observed in all abdominal interventions. This rate is consistent with the findings in the literature. Postoperative intraabdominal hemorrhages were related to improper and insufficient hemostasis in the initial surgery with a rate of $72.2 \%$ (28). In postoperative intra-abdominal hemorrhages, delayed diagnosis-related mortality rate was found to be $18.4-33.33 \%(7,29)$. In the study of Koirala $\mathrm{R}$ et al., intra-abdominal hemorrhage-related mortality rate was $42.4 \%$. This high mortality rate compared to the findings in the literature was suggested to be due to high-volumed liver and pancreas surgeries (28). The rate of intra-abdominal hemorrhage related mortality in our study was $9.3 \%$, and this was compatible with the findings in the literature. 
One of the important causes of UARLs is postoperative mechanical intestinal obstructions. It has been reported in different series that $5-60 \%$ of redolaparatomies were performed due to intestinal obstructions (2). This rate was $5 \%$ in the study of Unalp et al. and $6.7 \%$ in the study of Koirala R et al. $(1,2)$. Postoperative adhesions are the most important causes of obstructions (30). Other causes include intra-abdominal sepsis, abdominal dehiscence, previous femoral or other inguinal hernias (31). The frequency of mechanical intestinal obstructions developing in the early postoperative period is less than $1 \%$ (32). These patients may be recommended surgical decompression, nasogastric decompression or conservative approach (33). In our study, the rate of UARL due to mechanical intestinal obstruction was $13.5 \%$. This rate was consistent with the findings in the literature. The conservative approach or UARL for mechanical intestinal obstruction is still a debate. There is no consensus on the timing of re-laparatomy either. In the study of Unalp et al., the mean redolaparatomy interval was 4 days, whereas it was 12.7 days in the study of Koirala $\mathrm{R}$ et al. $(1,2)$. Median interval to first redo-laparatomy was $5(2,7)$ days in our study. The longer interval time may be primarily due the surgeons selecting a more conservative approach. The mortality rates in mechanical intestinal obstuction related redo-laparatomies was found to be approximately $10 \%$ (34). The mortality rates in patients undergoing redo-laparatomies due to mechanical intestinal obstructions was found to be as $14.2 \%$ in our study.

Different centers report different causes of mortality following UARLs. In the study of Koirala R et al., the most frequent causes of mortality were sepsis ve multi-organ failure in $64 \%$ of the patients. In the study of Oddeke VR, Haluk R and Wain MO, the sepsis and multi-organ failure were reported to be the most frequent cause of mortality as well $(1,22,31)$. Sepsis and multi-organ failure were the most frequent cause of mortality in our study as well, with a rate of $53.4 \%$. This result was consistent with the findings in the literature.

APACHE II reflects the Acute Physiology and Chronic Health situation, and helps evaluate the severity of the disease. There is a relationship between high score and mortality. In the study of Pusajó JF et al. on postoperative intra-abdominal sepsis requiring re-operation, the APACHE II score was found to be significantly higher in the mortality group (3). Hinsdale JG et al. have reported that patients undergoing re-laparatomy due to intra-abdominal sepsis and multi-organ failure demonstrated significantly higher mortality rates (8). Multi-organ failure was also high in the mortality group in our study. No significant relationship was found between the initial surgery performed under emergency conditions and presence of comormidity, and the mortality in patients undergoing UARL. This finding is consistent with the literature $(1,2)$.

We found a significant correlation between the blood transfusions and mortality in patients undergoing UARL. Blood transfusion may lead to coagulation disorders or DIC. The increased mortality in our study may be directly related to the complications of blood transfusion. On the other hand, it may be related to the acidosis that develope as a result of hemorrhage, and tissue hypoxia. Thus, blood transfusions may be considered as an indirect indicator of a patient whos is in a critical situation.

\section{Study limitations}

Although, there are a few limitations in our study, this research was a multi-center study and had rather wide series, more parameters could not be compared since it was a retrospective study. In order to understand the relationship between the surgeries and mortality, it may be necessary to evaluate each surgery separately. Initial UARLs, risk factors in UARLs performed for the second, third and more than three times, and indications at redo-laparotomy may be evaluated separately. While many operations were performed, the decision-making of UARLs may vary among the two centers, even if the same diagnostic tool was used. Factors affecting mortality following UARLs should be further investigated in prospective studies including larger series.

\section{Conclusion}

Sepsis and multi-organ failure were the most frequent cause of mortality in our study with a rate of $53.4 \%$. No significant relationship was observed between mortality and initial surgery under emergency conditions and presence of comorbidity. Redolaparatomies are inevitable in some clinical situations. Since multiple redolaparatomies are associated with mortality, the first redolaparatomy is very important. It is necessary to avoid complications during initial surgery to reduce UARLs and mortality. The surgeon should also make the UARLs decision at the right time.

Conflict of Interest: The authors declare no potential conflicts of interest with respect to the research, authorship, and/or publication of this article.

Author's Contributions: EU, SKT: Collecting of patients data, Patient examination and operation, writing and revision of article, EF: Statistical analysis of findings.

Ethical issues: All Authors declare that Originality of research/article etc... and ethical approval of research, and responsibilities of research against local ethics commission are under the Authors responsibilities. The study was conducted due to defined rules by the Local Ethics Commission guidelines and audits. 


\section{References}

1. Koirala R, Mehta N, Varma V, Kapoor S, Kumaran V, Nundy S. Urgent Redo-Laparotomies: Patterns and Outcome-A Single Centre Experience. Indian J Surg 2015;77(3):195-9.

2. Haluk RU, Erdinc K, Haldun K, Ahmet B, Mustafa P, Mehmet AO. Urgent abdominal re-explorations. World J Emerg Surg. 2006;1:10.

3. Pusajó JF, Bumaschny E, Doglio GR, et al. Postoperative intra-abdominal sepsis requiring reoperation. Value of a predictive index. Arch Surg 1993;128(2):218-22.

4. Fry DE, Garrison RN, Neitsch RC, Calhoun K, Polk HC Determinants of death in patients with intra-abdominal abscess. Surgery1980; 88(4):517-523.

5. Christou NV, Barie PS, Dellinger EP, Waymack JP, Harlan H. Surgical Infection Society Intra-abdominal Infection Study. Arch Surg 1993;128(2):193-199.

6. Butler JA, Huang J, Wilson SE. Repeated laparotomy for postoperative intra-abdominal sepsis. An analysis of outcome predictors. Arch Surg 1987;122(6):702-6.

7. Ching SS, Muralikrishnan VP, Whiteley GS Relaparotomy: a fiveyear review of indications and outcome. Int J Clin Pract 2003; 57(4):333-337.

8. Hinsdale JG, Jaffe BM. Re-operation for intra-abdominal sepsis. Indications and results in modern critical care setting. Ann Surg 1984;199(1):31-6.

9. Nakayama Y, Konishi M, Gotohda N, et al. Comparison of postoperative early and late complications between pancreas-sparing duodenectomy and pancreatoduodenectomy. Surg Today 2017;47(6):705-711.

10. Loveček $M$, Skalický $P$, Köcher $M$, et al Postpancreatectomy haemorrhage (PPH), prevalence, diagnosis and management. Rozhl Chir 2016;95(9):350357.

11. Teichmann W, Wittmann DH, Andreone PA. Scheduled reoperations (etappenlavage) for diffuse peritonitis. Arch Surg. 1986;121(2):147-52.

12. Sautner T, Gotzinger P, Redl-Wenzel EM, et al. Does reoperation for abdominal sepsis enhance the inflammatory host response? Arch Surg 1997;132(3):250-5

13. Wittmann DH, Aprahamian C, Bergstein JM. Planned relaparotomy: advanced diffuse peritonitis managed by planned multiple laparotomies utilizing zippers, slide fastener, and Velcro analogue for temporary abdominal closure. World J Surg. 1990;14(2):218-26.

14. Billing A, Frohlich D, Mialkowskyi O, Stokstad P, Schildberg FW. Treatment of peritonitis with staged lavage: prognostic criteria and course of treatment. Langenbecks Arch Chir 1992;377(5):305-13.

15. Nathens AB, Rotstein OD, Marshall JC. Tertiary peritonitis: clinical features of a complex nosocomial infection. World J Surg 1998;22(2):158-63.

16. Marshall JC, Christou NV, Horn R, Meakins JL. The microbiology of multiple organ failure. The proximal gastrointestinal tract as an occult reservoir of pathogens. Arch Surg 1988;123(3):309-15.

17. Mulier S, Penninckx F, Verwaest $\mathrm{C}$ et al. Factors affecting mortality in generalized postoperative peritonitis: multivariate analysis in 96 patients. World J Surg 2003;27(4):379-384.

18. Mulari K, Leppaniemi A. Severe secondary peritonitis following gastrointestinal tract perforation. Scand J Surg. 2004;93(3):204-208

19. Zavernyi LG, Poida AI, Melik VM, et al. Prognosis in the outcome of relaparotomy. Klin Khir 1992;(8):12-16

20. Oddeke VR, Cecilia WM, Kimberly RB. Comparison of on-demand vs planned relaparotomy strategy in patients with severe peritonitis: a randomized trial. JAMA 2007;298(8):865-872.

21. Holzheimer RG, Gathof B. Re-operation for complicated secondary peritonitis - how to identify patients at risk for persistent sepsis. Eur J Med Res 2003;8(3):125-134.

22. Hutchins RR, Gunning MP, Lucas DN, Allen-Mersh TG, Soni NC. Relaparotomy for suspected intraperitoneal sepsis after abdominal surgery. World J Surg 2004; 28(2):137-141

23. Rygachev GP, Nekhaev AN, Kerez PI, Kremen VE. Relaparotomy in the treatment of generalized postoperative Peritonitis. Khirurgiia 1997;(1):45-48.

24. Harbrecht PJ, Garrison RN, Fry DE. Early urgent relaparotomy. Arch Surg 1984; 119(4):369-374.

25. Tasu JP, Vesselle G, Herpe G, et al. Postoperative abdominal bleeding. Diagn Interv Imaging 2015;96(78):823-31.

26. Tera H, Aberg C. Relaparotomy. A ten-year series. Acta Chir Scand 1975;141(7):637-644

27. Krivitskii D, Shuliarenko VA, Babin IA. Indications for relaparotomy. Klin Khir 1990; (1):18-21.

28. Kononov AG, Sotnicenko BA, Makarov VI. Relaparotomy for intra-abdominal hemorrhage. Acta Chir Iugosl 1990;37(1):65-73.

29. Mamchich VI, Shaprinskii VA, Palienko PK. Intraabdominal hemorrhage after surgery on the abdominal organs requiring relaparotomy. Klin Khir 1992;(8):31-34.

30. Leshchenko IG, Panov FI. Relaparotomy for postoperative mechanical intestinal obstruction in abdominal injuries. Vestn Khir Im I I Grek 1991;146(4):88-91.

31. Wain MO, Sykes PA. Emergency abdominal re-exploration in a district general hospital. Ann R Coll Surg Engl. 1987;69(4):169-74

32. Zavernyi LG, Poida AI, Tarasov AA, Mel'nik VM, Nadeev SS. Indications for relaparotomy in acute postoperative intestinal obstruction. Klin Khir 1992; (4):4-7.

33. Ellozy SH, Harris MT, Bauer JJ, Gorfine SR, Kreel I. Early postoperative small-bowel obstruction: a prospective evaluation in 242 consecutive abdominal operations. Dis Colon Rectum 2002;45(9):1214-1217.

34. QuatromoniJC, RosofiL, HallsJ, Yellin AE. Early postoperative small bowel obstruction. Ann Surg1980; 191(1):72-4.

Copyright (C) 2016 The Author(s); This is an open-access article distributed under the terms of the Creative Commons Attribution License (http://creativecommons.org/licenses/by/4.0), which permits unrestricted use, distribution, and reproduction in any medium, provided the original work is properly cited. All Rights reserved by international journal of Medical Science and Discovery. 